\title{
Same-Session Bilateral Retrograde Intrarenal Surgery for Upper Urinary System Stones: Safety and Efficacy
}

\author{
Erdal Alkan, MD, Egemen Avci, MD, ${ }^{2}$ Ahmet Oguz Ozkanli, MD, ${ }^{3}$ Oguz Acar, MD, \\ and Mevlana Derya Balbay, MD ${ }^{1}$
}

\begin{abstract}
Purpose: We present our experience with same-session retrograde intrarenal surgery (RIRS) for the management of bilateral upper urinary system stones (UUSS) in terms of clearance of stones, operative times, duration of hospital stay, and complications.

Materials and Methods: Between November 2007 and January 2013, a total of 44 simultaneous bilateral RIRS were performed at our hospital. Initially, symptomatic sides of the patients were operated on, and, when asymptomatic, the greater stone burden was treated first. Having completed stone fragmentation with a holmium:YAG laser, larger fragments were extracted with a nitinol basket. All patients underwent noncontrast CT scanning or urinary ultrasonography 2 months after the removal of the stent to detect any residual fragments. The stone-free status was defined as no fragments and/or the presence of asymptomatic fragments $<4 \mathrm{~mm}$ in the urinary system. Results: The total stone number was 201 with a mean stone burden per patient of $30.0 \pm 15.4 \mathrm{~mm}$ (range 10$85 \mathrm{~mm}$ ). The overall stone-free rate (SFR) was $88.6 \%$ after all procedures. The patients in this study were divided into two groups according to stone burden: Although the overall SFR was $100 \%$ for a stone burden $<25 \mathrm{~mm}$, the SFR was $80 \%$ for a stone burden $\geq 25 \mathrm{~mm}(P=0.006)$. A Double-J stent was not placed in two patients, and they developed postoperative anuria; both cases were treated with Double-J stent placement. Conclusion: Bilateral same-session RIRS is a safe and effective procedure that can be considered a first-line treatment for bilateral UUSS in select patients. The SFR is satisfactory, especially in patients with a stone burden $<25 \mathrm{~mm}$. At minimum a unilateral Double-J stent should be placed in patients undergoing bilateral RIRS to avoid postrenal failure.
\end{abstract}

\section{Introduction}

$\mathbf{T}$ HERE ARE VARIOUS TREATMENT OPTIONS for upper urinary system stones (UUSS); they include extracorporeal shockwave lithotripsy (SWL), ureterorenoscopy (URS), percutanous nephrolithotomy (PNL), and open surgery. Multiple stones are found in $20 \%$ to $25 \%$ of patients with urolithiasis. ${ }^{1,2}$ The stone-free rates (SFRs) for patients with multiple stones after SWL are reportedly $40 \%$ to $50 \%$, which is significantly lower than those for patients with a single stone. ${ }^{1}$ The superiority of URS over SWL is its ability to directly access stones throughout the entire urinary system either unilaterally or bilaterally and to actively remove stone fragments. Conversely, PNL is currently the first-line standard procedure for patients with renal stones $>20 \mathrm{~mm}$, multiple renal stones, or stones located in the lower pole area. Although PNL is more effective than URS or SWL in the treatment of kidney stones, it is more invasive and has higher morbidity rates compared with URS or SWL. ${ }^{3}$ When the stone number increases and stone size decreases, the SFR will dramatically decrease after PNL.

With recent developments in endoscopic technology, such as smaller caliber flexible ureteroscopes, dual deflection, improved optics, use of holmium lasers, ureteral access sheaths, and new generation stone extractors, ureterorenoscopic procedures are becoming more common. Urologists can now reach stones virtually anywhere in the upper urinary tract with minimal difficulty. There are few reports in the literature about same-session retrograde intrarenal surgery (RIRS) for the management of bilateral UUSS. $^{4-6} \mathrm{~A}$ reduction in overall operative time, anesthetic requirements, and duration of convalescence are some of the advantages of same-session bilateral RIRS; conversely, the technique exposes both ureters to injury that could lead to significant morbidity. We believe that

Departments of Urology ${ }^{1}$ and Anesthesiology, ${ }^{3}$ Memorial Istanbul Sisli Hospital, Istanbul, Turkey.

${ }^{2}$ Department of Urology, Memorial Istanbul Atasehir Hospital, Istanbul, Turkey. 
bilateral RIRS may be a successful treatment in patients with bilateral UUSS and may demonstrate a valuable alternative to PNL and SWL in select patients.

In this study, we present our experience with same-session RIRS for the management of bilateral UUSS in terms of clearance of stones, operative times, duration of hospital stay, and complications.

\section{Patients and Methods}

\section{Patients}

Between November 2007 and January 2013, 42 patients with bilateral UUSS underwent simultaneous bilateral RIRS by three surgeons at our hospital. A total of 44 simultaneous bilateral RIRS were performed on the 42 patients ( 28 men and 14 women). Mean patient age was $40.1 \pm 10.8$ years (range $18-63$ ) and mean body mass index was $30.6 \pm 6.0 \mathrm{~kg} / \mathrm{m}^{2}$ (range 21-52). Two obese patients were treated with a separate additional RIRS session for residual fragments.

All patients were evaluated preoperatively by noncontrast CT scan with stone protocol to assess collecting system anatomy, total stone number, and stone burden, which was calculated by measuring the maximum stone dimension. In cases of multiple stones, these dimensions were added together. The indications for simultaneous bilateral RIRS were done in select cases; these included 1) failed SWL or PNL, 2) patients with concomitantly unilateral or bilateral ureteral and/or renal stones, 3) patients who required minimally invasive surgery (e.g., cases of coagulopathy or obesity), 4) patients with bilateral multiple stones $<25 \mathrm{~mm}$ for each stone, and 5) patient preferences. While bilateral flexible ureteroscopy was performed in all cases, a rigid ureteroscope was additionally used in some patients with lower or middle ureteral stones. Each patient was evaluated for the operative time, SFR, duration of hospital stay, and perioperative complications. If required, serum creatinine was measured in the preoperative and postoperative periods to evaluate postoperative renal function. Patient follow-up was continued at least 3 months after surgery to evaluate any complications and assess the SFR.

\section{Technique}

Preoperatively all patients were given intravenous antibiotics, usually first-generation cephalosporin, unless preoperative urine culture suggested a different antibiotic. According to availability, the URF P-5 flexible ureteroscope (Olympus, Tokyo, Japan) or Cobra flexible dual-channel ureteroscope (Wolf, Germany) were used through a ureteral access sheath (Flexor ureteral access sheath 12F/14F $35 \mathrm{~cm}$; Cook Medical, Bloomington, IN). Symptomatic sides of the patients were initially operated on; when asymptomatic, greater stone burden was treated first. In case the ureteral access sheath could not be advanced unilaterally or bilaterally due to ureteral stricture, a $4 \mathrm{~F} / 8 \mathrm{~F} 26 \mathrm{~cm}$ Double-J stent was inserted into the ureter bilaterally, and the procedure was delayed at least 15 days. If a complication occurred, the procedure was cancelled. In this study, patients on whom bilateral flexible ureteroscopy (FURS) could not have been done were excluded.

The Holmium:YAG laser (LISA Sphinx 20W, Katlenburg, Germany), with a 200 to $270 \mathrm{~m}$ fiber, was set at an energy level of 0.6 to $1.4 \mathrm{~J}$ and a rate of 5 to $8 \mathrm{~Hz}$; it was applied to fragment stones into pieces $<4 \mathrm{~mm}$ in its largest diameter. Larger stone fragments were extracted with a nitinol basket (NGage Nitinol stone extractor 2,2F $115 \mathrm{~cm}$ basket; Cook Medical). Endoscopically, intraoperative success was defined as extraction of all stone fragments or laser lithotripsy of all stones to $<4 \mathrm{~mm}$ fragments.

At the end of the operation, a Double-J stent was placed unilaterally, bilaterally, or not at all at the surgeon's discretion depending on stone burden and length of the procedure. Residual fragments and the position of the Double-J stent in the urinary system were assessed with plain radiographs on postoperative day 1 . Patients who had residual fragments $>4 \mathrm{~mm}$ were recommended for an additional operation in approximately 4 weeks after the first operation. The Double-J stents were removed under local anesthesia by flexible cystoscope 3 to 4 weeks after the procedure when the patient was rendered stone-free bilaterally or refused to continue any intervention.

All patients underwent noncontrast CT scanning or urinary ultrasonography 2 months after the removal of the Double-J stent to detect any residual fragments. Stone-free status was defined as no fragments and/or the presence of asymptomatic fragments $<4 \mathrm{~mm}$ in the urinary system. Intraoperative and postoperative complications associated with same-session bilateral RIRS were recorded.

\section{Statistical methods}

Statistical analysis was performed using SPSS 16.0 for Windows software (SPSS, Inc., Chicago, IL). A comparison between the groups was done by using Student t-test and Pearson $\chi^{2}$-test. The difference was considered statistically significant at $<0.05$.

\section{Results}

Stones characteristics are shown in Table 1. All patients were divided into two groups according to stone number

\section{Table 1. Stone Characteristics}

\begin{tabular}{lc}
\hline Mean stone size $(\mathrm{mm}):$ & $6.4 \pm 4.1(2-25)$ \\
Right side & $6.4 \pm 4.0(2-25)$ \\
Left side & $6.4 \pm 4.2(2-25)$ \\
Mean stone burden $(\mathrm{mm}):$ & $30.0 \pm 15.4(10-85)$ \\
Right side & $15.5 \pm 9.2(3-50)$ \\
Left side & $14.5 \pm 8.3(4-40)$ \\
Mean stone number per patient $(n):$ & $4.7 \pm 1.9(2-11)$ \\
Right side & $2.4 \pm 0.9(1-5)$ \\
Left side & $2.2 \pm 1.2(1-6)$ \\
Total stone number $(n):$ & 201 \\
Right side & 107 \\
Left side & 94 \\
Stone location $(n, \%):$ & $32(16 \%)$ \\
Upper calyx & $39(19 \%)$ \\
Middle calyx & $53(26 \%)$ \\
Lower calyx & $35(17 \%)$ \\
Renal pelvis & $23(12 \%)$ \\
Upper ureter & $6(3 \%)$ \\
Middle ureter & $13(7 \%)$ \\
Lower ureter & \\
Distribution of the stones $(n, \%):$ & $88(100 \%)$ \\
Total renal unit & $47(53.5 \%)$ \\
Renal only & $37(42 \%)$ \\
Renal and ureteral & $4(4.5 \%)$ \\
Ureteral only &
\end{tabular}


Table 2. Operative Data on Patients Segregated by Stone Number $(>5 v \leq 5)$

\begin{tabular}{lccc}
\hline & $>5$ stones & $\leq 5$ stones & P value \\
\hline Number of procedures $(n, \%)$ & $27(61.4 \%)$ & $17(38.6 \%)$ & \\
Stone burden (mm, range) & $26.5 \pm 15.1(10-85)$ & $35.0 \pm 15.1(20-75)$ & 0.001 \\
Mean stone number $(n$, range) & $3.4 \pm 0.6(2-4)$ & $6.7 \pm 1.5(5-11)$ & 0.001 \\
Mean operation time (min, range) & $84.4 \pm 38.1(35-180)$ & $96.5 \pm 31.1(45-175)$ & 0.404 \\
Overall SFR & $92.6 \%(25 / 27)$ & $82.3 \%(14 / 17)$ & 0.433 \\
\hline
\end{tabular}

$\min =$ minutes $; \mathrm{n}=$ number; $\mathrm{SFR}=$ stone-free rate.

(Table 2) and stone burden (Table 3). In six patients with bilateral ureteral stones, preoperative serum creatinine levels were recorded (mean $2.1 \mathrm{mg} / \mathrm{dL}$, range $0.8-2.9 \mathrm{mg} / \mathrm{dL}$ ). Rigid ureteroscope was used in 17 (19\%) ureteral units for concomitant lower or middle ureteral stones (a total of 19 stones) in addition to bilateral UUSS treated with bilateral flexible ureteroscopy.

Bilateral RIRS was done for $8(18 \%)$ and $3(7 \%)$ patients due to failed SWL and failed PNL, respectively. Bilateral ureteral stones, together with renal stones, were present in 6 (14\%) patients who were treated in the emergency setting. For the 13 (34\%) of 42 patients (15 procedures) who were obese or morbidly obese, no treatment was recommended except RIRS. In 3 of the 13 obese or morbidly obese patients, the stone size was $>20 \mathrm{~mm}$, and these patients did not undergo bilateral PNL because of our surgical inexperience in obese patients. Bilateral RIRS was done in $3(7 \%)$ patients with coagulopathy and $9(20 \%)$ patients because of patient preferences (two were pilots and one worked in shipping).

Four stones were found in one patient $(20 \mathrm{~mm}$ in the right upper ureter, $25 \mathrm{~mm}$ in the right lower calyx, $25 \mathrm{~mm}$ in the left upper ureter, $15 \mathrm{~mm}$ in the left lower calyx), with a total stone burden of $85 \mathrm{~mm}$. The preoperative serum creatinine level was $2.9 \mathrm{mg} / \mathrm{dL}$. As a result, bilateral RIRS was planned in the emergency setting, and the operative time was 180 minutes. SWL or second-session RIRS was proposed following bilateral RIRS due to bilateral residual stone fragmentations, with the largest sizes $7 \mathrm{~mm}$ and $6 \mathrm{~mm}$ bilaterally, but the patient refused further treatment.

Mean operative time and mean duration of hospital stay were $89.1 \pm 35.7$ minutes (range 35-180) and 29.5 \pm 13.6 hours (range 3-72), respectively. The operative time was 35 minutes in one patient who was discharged at 3 hours after the operation. Although we insisted he stay at least 6 hours, the patient refused to remain in the hospital. At the end of the session, 32 of 44 procedures (73\%) were judged endoscopically stone-free bilaterally, 8 procedures $(18 \%)$ were judged stone-free unilaterally with residual stones $>4 \mathrm{~mm}$ in the contralateral system, and the remaining 4 cases (9\%) were assessed as not stone-free bilaterally. Those patients assumed to be stone-free intraoperatively were evaluated by ultrasonography, while patients with residual stone burden observed during intraoperative evaluation were examined by noncontrast CT scanning 2 months after removal of the Double-J stent. The ureteral access sheath could not be advanced in two patients bilaterally and three patients unilaterally at the outset of the procedure. These patients were stented bilaterally, and after 15 days bilateral RIRS was done easily on all of them.

Thirty-eight patients (90\%) were stone free after only one RIRS session. Another patient who was morbidly obese (2.4\%) required a second session of RIRS and become stone free. Another patient who was obese also required a second RIRS for residual fragmentations bilaterally and could not be rendered stone free. This patient and two other patients with unilateral residual stones after first procedure were recommended for second-session RIRS or SWL, but they refused further treatments. The overall SFR was $88.6 \%$ after all procedures.

All patients were treated with holmium lasers and basket catheters, except $11(25 \%)$ patients who were treated with lasers only. At the end of the procedure, Double-J stents were placed in 36 patients (82\%), bilaterally in $33(75 \%)$ and unilaterally in $3(7 \%)$. Double-J stents were not placed, however, in $8(18 \%)$ patients; two $(4.5 \%)$ of these patients developed postoperative anuria and subsequent azotemia on postoperative day 1 due to bilateral ureteral edema. Except for these two cases, there were no major perioperative complications.

Minor complications occurred in $5(11.4 \%)$ patients; these complications included prolonged hematuria in $3(6.8 \%)$ cases and urinary tract infection treated with antibiotics in 2 (4.6\%) cases. Additionally, 5 (11.4\%) patients were readmitted to the emergency room because of renal colic and treated with parenteral medications in outpatient settings. Grade II ureteral injury was seen intraoperatively in 8 (9.1\%) ureters due to ureteral access sheath and other instruments, according to Traxer and associates. ${ }^{7}$ The procedures were not cancelled in any of these patients, and none required further treatment other than Double-J stent placement.

Stone composition analyses were done in 20 patients. Calcium oxalate monohydrate and dehydrate, mixed (calcium oxalate monohydrate, dehydrate, and calcium phosphate),

Table 3. Operative Data on Patients Segregated by Stone Burden ( $<25$ mm $v \geq 25$ mm)

\begin{tabular}{lccr}
\hline & Stone burden $<25 \mathrm{~mm}$ & Stone burden $\geq 25 \mathrm{~mm}$ & P value \\
\hline Number of procedures $(n, \%)$ & $19(43 \%)$ & $25(57 \%)$ & \\
Stone burden (mm, range) & $18.4 \pm 3.5(10-24)$ & $38.7 \pm 16.4(25-85)$ & 0.001 \\
Mean stone number $(n$, range) & $3.6 \pm 1.2(2-7)$ & $5.5 \pm 2.0(3-11)$ & 0.001 \\
Mean operative time (min, range) & $61.3 \pm 21.3(35-135)$ & $110.2 \pm 29.1(45-180)$ & 0.001 \\
Overall SFR & $100 \%(19 / 19)$ & $80 \%(20 / 25)$ & 0.006 \\
\hline
\end{tabular}

$\min =$ minutes $; \mathrm{n}=$ number; $\mathrm{SFR}=$ stone-free rate. 
magnesium ammonium phosphate, and uric acid stones were seen in $10(50 \%), 7(35 \%), 2(10 \%)$, and $1(5 \%)$ of cases, respectively.

\section{Discussion}

There is no consensus in the management of patients with bilateral UUSS, and optimal treatment for these stones remains controversial. Patients with bilateral UUSS can be treated mainly with a staged RIRS, but some can also be treated with simultaneous RIRS. There have been some reports of same-session bilateral SWL, PNL, and RIRS. ${ }^{4-6,8-11}$

According to American Urological Association (AUA) and European Association of Urology (EAU) guidelines, SWL is the first-line treatment for intrarenal stones $<20 \mathrm{~mm}^{12,13}$ Certain indications may preclude the use of SWL, however; these include pregnancy, morbid obesity, coagulopathy, and some intrinsic and extrinsic anatomic problems (e.g., patients with extremity deformity, the existence of ureteral strictures). Although the SFR range is $80 \%$ to $88 \%$ in the management of UUSS with SWL, it decreases to $40 \%$ to $50 \%$ in patients with multiple intrarenal and lower calyx stones. ${ }^{1-3}$ It can also be applied bilaterally. ${ }^{8,9}$ Perry and associates evaluated 120 patients who had undergone bilateral same-session SWL and reported that bilateral SFR after a single session without major complication was $60 \%$ and $16 \%$ of the patients required additional procedures for residual particles. 9 Although SWL has fewer complications compared with both URS and PNL, the stone street formation, retroperitoneal hematoma, and renal injuries cannot be ignored. ${ }^{14}$

PNL, according to AUA and EAU guidelines, is the firstline treatment for intrarenal stones $>20 \mathrm{~mm} .{ }^{12,13} \mathrm{PNL}$ is the most invasive treatment modalities for kidney stones and has an excellent SFR of $86 \%$ to $100 \% .^{3}$ Because of its invasiveness, it has some serious complications, however, which arise from percutaneous access. The complication rates include $7.8 \%$ hemorrhage, $3.4 \%$ renal collecting system perforation, and $1.8 \%$ hydrothorax. ${ }^{15}$ Same-session PNL can be performed bilaterally in patients with bilateral UUSS, ${ }^{10,11}$ especially when the stone burden is not very large. However, bilateral interventions cannot be handled in a single session at times, mostly due to multiple accesses, and complication rates may increase. ${ }^{16}$

The EAU 2013 urolithiasis guidelines do not recommended RIRS as first-line treatment, especially for stones $>1.5 \mathrm{~cm}$ in the renal pelvis and upper or middle calices, for which SFR after RIRS decreases and staged procedures become necessary. ${ }^{12}$ RIRS can be successful with experienced surgeons in high-volume centers, however. ${ }^{12}$ Conversely, PNL or RIRS are recommended for lower calyx stones, even for stones $>1.5 \mathrm{~cm}$, because the SWL efficacy is limited. ${ }^{12}$ With the recent advancements in endoscopic technology, including flexible ureteroscopes (especially digital ureteroscopes), laser lithotriptors, and smaller caliber instruments (access sheaths, baskets, and laser fibers), RIRS yielded similar SFR and lower complication rates compared with PNL and higher success rates compared with SWL, and RIRS has been more popular than either of these procedures. ${ }^{6}$ Stones anywhere in the upper urinary tract can now be reached and treated with RIRS with minimal difficulty.

Although there are several studies related to bilateral rigid ureteroscopy for patients with bilateral ureteral stones, ${ }^{17,18}$ there are few reports in the literature about same-session RIRS for the management of bilateral UUSS. ${ }^{4-6}$ Chon and associates reported bilateral RIRS for the first time in 2005. ${ }^{19}$ There have been two major studies about bilateral RIRS for patients with bilateral UUSS. ${ }^{5,6}$ Huang et al performed bilateral RIRS in 25 patients with bilateral renal stones and reported overall SFR of $70 \%$ after a single procedure and $92 \%$ after second and third procedures. ${ }^{5}$ The patients in this study were divided into two groups according to stone burden. While the overall SFR was $100 \%$ for stone burden $\leq 20 \mathrm{~mm}$, the SFR was $85.7 \%$ for stone burden $>20 \mathrm{~mm}$ after all treatment. In another study, Atis et al. reported 42 patients who underwent same-session bilateral RIRS for bilateral UUSS. In their study, the SFRs were $92.8 \%$ and $97.2 \%$ after the first and second procedures, respectively. ${ }^{6}$ In the present study, 44 same-sessions bilateral RIRSs were performed on 42 patients, and overall SFRs were $88.6 \%$ after all procedures, compatible with the current literature. The SFR for bilateral RIRS is similar to that reported for bilateral PNL ${ }^{10,11}$ and is better than rates reported for bilateral EWSL. ${ }^{8,9}$

We divided our patients into two groups according to stone burden $(<25 \mathrm{~mm} v \geq 25 \mathrm{~mm})$ and stone number $(>5 v \leq 5)$. Same-session bilateral RIRS was performed in our study for the following reasons: obesity (34\%); insufficient treatment modalities, such as SWL or PNL (25\%); unilateral or bilateral ureteral stones requiring emergency treatment (14\%); patient preferences (20\%); and coagulopathy (7\%). In our series, there were indications of bilateral PNL in five patients, three of whom were obese. Bilateral PNL was not performed because of surgical inexperience. The other two patients preferred bilateral RIRS to PNL.

Overall SFR was $100 \%$ for stone burden $<25 \mathrm{~mm}$ and the SFR was $80 \%$ for stone burden $\geq 25 \mathrm{~mm}(P=0.006)$ in our series. These results were statistically significant and compatible with the literature. Similarly, the overall SFR were $92.6 \%$ and $82.3 \%$ for stone numbers $>5$ and $\leq 5$, respectively $(P=0.433)$. To our knowledge there has been no previous study where SFR and stone numbers were correlated in patients who underwent bilateral RIRS. We did not find any correlation between SFR and stone number. Mean stone burden and mean operative times were the other important parameters for patients who underwent bilateral RIRS. In the studies mentioned above, mean stone burden and mean operative times were $24 \pm 5 \mathrm{~mm}$ and $81.2 \pm 25$ minutes $^{5}$ and $24.09 \pm 6.37 \mathrm{~mm}$ and $51.08 \pm 15.22$ minutes, ${ }^{6}$ respectively. In our study, mean stone burden and mean operative time were $30.0 \pm 15.4 \mathrm{~mm}$ and $89.1 \pm 35.7$ minutes, respectively. Mean operative time was longer than those reported in other studies due to the use of nitinol basket and larger stone burden. Additionally, the mean operative time for bilateral RIRS was found to be shorter than that reported for PNL in the literature. $^{20}$

Ureterorenoscopic interventions may cause some complications, such as ureteral avulsion, perforation, false passage, rupture of balloon dilator, ureteral edemas, access problems, urinomas, ureteral strictures, stone migration, urinary tract infections, and urosepsis. ${ }^{12,21}$ These are more important in bilateral cases. There are different complication rates related to bilateral URS in the literature, including urosepsis, pulmonary embolus resulting in death, ${ }^{22}$ hematuria and urinary system infection, ${ }^{5}$ postoperative fever, and prolonged hematuria. ${ }^{6}$ Traxer and colleagues studied 359 consecutive 
patients who underwent RIRS for kidney stone. ${ }^{7}$ In their study, ureteral wall injury was found in 167 patients $(46.5 \%)$. There were grade II, III, and IV injuries in $10.1 \%, 3.3 \%$, and $0.0 \%$ of patients, respectively. In the current study, grade II ureteral injury as described by Traxer was seen in eight patients $(9.1 \%)$ intraoperatively. ${ }^{7}$ None of the procedures were canceled, and none required further treatment other than Double-J stent placement. No long-term complication was seen in these patients. Because the small mucosal erosions were ignored in the ureter, ureteral injury rates were lower than those reported by Traxer et al. In our study, minor postoperative complications were encountered in five patients $(11.4 \%)$ : prolonged hematuria in three cases that resolved spontaneously and urinary tract infection in two cases treated with intravenous antibiotics. Additionally, five patients $(11.4 \%)$ were readmitted to the emergency room because of renal colic and treated with parenteral medications in an outpatient setting.

Although several randomized prospective studies have recommended that Double-J stent placement is not routinely necessary following uncomplicated URS, most urologists routinely insert a Double-J stent after URS. ${ }^{12,23}$ According to EAU 2013 guidelines, stents should be inserted in patients who are at increased risk of complications, such as residual fragments, bleeding, perforation, pregnancy, and infections. ${ }^{12}$ Bilateral ureteral edema resulting in acute renal failure may develop following bilateral RIRS. ${ }^{24}$ In our study, the ratio of Double-J stent placement was $82 \%$ (bilaterally in $75 \%$ and unilaterally in $7 \%$ ). Stents were not placed in eight cases considered uncomplicated. Of these, two patients developed anuria on postoperative day 1, and Double-J stents were placed bilaterally in both cases. Postoperative anuria has not been reported in the previously published studies because a Double-J stent was placed at least unilaterally. ${ }^{4-6}$ In light of our findings, we now place at least a unilateral stent in patients undergoing bilateral RIRS to prevent postoperative anuria.

Our study has some limitations. The most important limitation is its retrospective nature. Another potential limitation is the small number of patients treated with bilateral RIRS. Larger studies with longer follow-up times are necessary to confirm the long-term value of same-session bilateral RIRS. Also, the interventions were performed by three surgeons, and reporting on a patient series treated by a single surgeon would prevent bias and differences between surgeons. In our study, however, this would have resulted in a smaller series. We feel that combining results of three surgeons not only resulted in a larger series but also reflected more realistic results that practicing urologists can adopt. In spite of these limitations, our study is one of the largest series in the literature that proves bilateral RIRS is technically feasible and safe procedure.

\section{Conclusion}

Bilateral same-session RIRS is a safe and effective procedure and can be considered as a first-line treatment for bilateral UUSS in select patients. Its advantages include a short hospital stay, prevention of multiple procedures, reduced anesthetic needs, and reduced overall costs. The SFR is satisfactory, especially in patients with a stone burden $<25 \mathrm{~mm}$. We recommend that at least a unilateral Double-J stent should be placed in patients undergoing bilateral RIRS to avoid postrenal failure.

\section{Disclosure Statement}

No competing financial interests exist.

\section{References}

1. Abe T, Akakura K, Kawaguchi M, et al. Outcomes of shockwave lithotripsy for upper urinary tract stones: A large-scale study at a single institution. J Endourol 2005;19: 768-773.

2. Kanao K, Nakashima J, Nakagawa K, et al. Preoperative nomograms for predicting stone-free rate after extracorporeal shock wave lithotripsy. J Urol 2006;176:1453-1457.

3. Galvin DJ, Pearle MS. The contemporary management of renal and ureteric calculi. BJU Int 2006;98:1283-1288.

4. Watson JM, Chang C, Pattaras JG, et al. Same session bilateral ureteroscopy is safe and efficacious. J Urol 2011; 185:170-174.

5. Huang Z, Fu F, Zhong Z, et al. Flexible ureteroscopy and laser lithotripsy for bilateral multiple intrarenal stones: Is this a valuable choice? Urology 2012;80:800-804.

6. Atis G, Koyuncu H, Gurbuz C, et al. Bilateral single-session retrograde intrarenal surgery for the treatment of bilateral renal stones. Int Braz J Urol 2013;39: 387-392.

7. Traxer O, Thomas A. Prospective evaluation and classification of ureteral wall injuries resulting from insertion of a ureteral access sheath during retrograde intrarenal surgery. J Urol 2013;189:580-584.

8. Pienkny AJ, Streem SB: Simultaneous versus staged bilateral extracorporeal shock wave lithotripsy: Long-term effect on renal function. J Urol 1999;162:1591-1593.

9. Perry KT, Smith ND, Weiser AC, et al. The efficacy and safety of synchronous bilateral extracorporeal shock wave lithotripsy. J Urol 2000;164:644-647.

10. Bagrodia A, Raman JD, Bensalah K, Pearle MS, Lotan Y. Synchronous bilateral percutaneous nephrostolithotomy: Analysis of clinical outcomes, cost and surgeon reimbursement. J Urol 2009;181:149-153.

11. Ugras MY, Gedik E, Gunes A, et al. Some criteria to attempt second side safely in planned bilateral simultaneous percutaneous nephrolithotomy. Urology 2008;72:996-1000.

12. Türk C, Knoll T, Petrik A, Sarica K, Straub M, Seitz C. Guidelines on Urolithiasis. www.uroweb.org/gls/pdf/18 Urolithiasis.pdf European Association of Urology, updated March 2011. Accessed: November 30, 2013.

13. Preminger GM. Management of lower pole renal calculi: Shock wave lithotripsy versus percutaneous nephrolithotomy versus flexible ureteroscopy. Urol Res 2006;34: 108-111.

14. Breda A, Ogunyemi O, Leppert JT, et al. Flexible ureteroscopy and laser lithotripsy for multiple unilateral intrarenal stones. Eur Urol 2009;55:1190-1196.

15. de la Rosette J, Assimos D, Desai M, et al. The Clinical Research Office of the Endourological Society Percutaneous Nephrolithotomy Global Study: Indications, complications, and outcomes in 5803 patients. J Endourol 2011;25:11-17.

16. Marguet CG, Springhart WP, Tan YH, et al. Simultaneous combined use of flexible ureteroscopy and percutaneous nephrolithotomy to reduce the number of access tracts in the management of complex renal calculi. BJU Int 2005; 96:1097-1100. 
17. Isen K. Single-session ureteroscopic pneumatic lithotripsy for the management of bilateral ureteric stones. Int Braz J Urol 2012;38:63-68.

18. Gunlusoy B, Degirmenci T, Arslan M, et al. Bilateral single-session ureteroscopy with pneumatic lithotripsy for bilateral ureter stones: Feasible and safe. Urol Int 2008; 81:202-205.

19. Chon $\mathrm{CH}$, Chung SY, Ng CS, Fuchs GJ. Simultaneous bilateral retrograde intrarenal surgery for bilateral complex upper tract stone disease. Urology 2005;65: $572-574$.

20. Mason BM, Koi PT, Hafron J, Milhoua P, Hoenig DM. Safety and efficacy of synchronous percutaneous nephrostolithotomy and contralateral ureterorenoscopy for bilateral calculi. J Endourol 2008;22:889-893.

21. Parker BD, Frederick RW, Reilly TP, Lowry PS, Bird ET. Efficiency and cost of treating proximal ureteral stones: Shock wave lithotripsy versus ureteroscopy plus holmium: yttrium-aluminum-garnet laser. Urology 2004;64:11021106.

22. Hollenbeck BK, Schuster TG, Faerber GJ, Wolf JS. Safety and efficacy of same-session bilateral ureteroscopy. J Endourol 2003; 17:881-885.

23. Nabi G, Cook J, N'Dow J, McClinton S. Outcomes of stenting after uncomplicated ureteroscopy: Systematic review and meta-analysis. BMJ 2007;334:572.
24. Bandi G, Vicentini FC, Triest JA. Anuric renal failure after same-session bilateral atraumatic flexible ureteroscopy. Int Braz J Urol 2007;33:193-194.

\section{Abbreviations Used}

AUA $=$ American Urological Association

$\mathrm{CT}=$ computed tomography

$\mathrm{EAU}=$ European Association of Urology

$\mathrm{PNL}=$ percutaneous nephrolithotomy

$\mathrm{RIRS}=$ retrograde intrarenal surgery

$\mathrm{SFR}=$ stone-free rate

$\mathrm{SWL}=$ extracorporeal shockwave lithotripsy

$\mathrm{URS}=$ ureterorenoscopy

UUSS $=$ upper urinary system stones

Address correspondence to:

Erdal Alkan, MD

Department of Urology

Memorial Istanbul Sisli Hospital

Okmeydani 34385

Sisli-Istanbul

Turkey

E-mail: eralkan@hotmail.com 